\title{
Permanent Magnet Temperature Estimation in PM Synchronous Motors Using Low Cost Hall Effect Sensors
}

\author{
Daniel Fernandez ${ }^{*}$, Doosoo Hyun ${ }^{* *}$, Yonghyun Park ${ }^{* *}$, David Reigosa*, Sang Bin Lee ${ }^{* *}$, Dong Myung Lee ${ }^{* * *}$ \\ and Fernando Briz ${ }^{*}$ \\ *Department of Electrical Engineering, \\ University of Oviedo, Spain \\ ${ }^{* *}$ Department of Electrical Engineering, \\ Korea University, Seoul, Korea \\ Email: sangbinlee@korea.ac.kr \\ ${ }^{* * *}$ School of Electronic and Electrical \\ Engineering, \\ Hongik University, Seoul, Korea
}

\begin{abstract}
Knowledge of the permanent magnet (PM) temperature in PM synchronous machines (PMSMs) is of great importance both for control and monitoring purposes. Increase in PM temperature during motor operation can degrade the magnetic flux strength and consequently the machine's torque production capability, also can cause irreversible demagnetization of the PM. Direct measurement of the PM temperature is not viable in practice, due to both cost and reliability issues. Indirect PM temperature estimation methods recently studied can require knowledge of thermal or electrical model parameters or can have undesired effects on motor operation. In this paper, the feasibility of using low cost halleffect sensors for PM temperature estimation is investigated. Hall sensors are present for detecting the initial position of the rotor in majority of PMSM applications for which incremental encoders are used for control. The proposed method can therefore be implemented with low or no additional cost. Experimental results on two IPMSMs show that the method is capable of providing non-invasive estimation of the PM temperature without a priori motor parameter information for monitoring and protection against excessive increase in temperature. ${ }^{1}$
\end{abstract}

Abstract-Demagnetization, Hall-effect sensor, Permanent magnet, PM synchronous motor, Thermal monitoring.

\section{INTRODUCTION}

PM temperature estimation in PMSMs is of great importance both for control and monitoring purposes [1]-[10]. For NdFeB magnet material, increase of the PM temperature reduces the PM strength and consequently degrades the torque production capability and operating efficiency of the machine. Furthermore, irreversible demagnetization can be caused by excessive increase in PM temperature or a combination of increase in PM temperature and demagnetizing armature magneto-motive force [10]-[11], [14]. Therefore, it is important to monitor the temperature of the PM to avoid operation under excessive temperatures to prevent degradation in performance, efficiency and reliability of the machine.

Direct measurement of the PM temperature is not a trivial task. Contact-type temperature sensors such as thermistors or

\footnotetext{
${ }^{1}$ This work was supported in part by the Regional Ministry of Education, Culture and Sport of the Principality of Asturias through "Severo Ochoa Program" under Grant BP-13067, and in part by the "Human Resources program in Energy Technology" of the Korea Institute of Energy Technology Evaluation and Planning (KETEP) granted financial resource from the Ministry of Trade, Industry \& Energy, Republic of Korea (No. 20154030200610)
}

thermocouples would require cabling to a rotating part through slip rings and brushes, or the use of a wireless transmission system [9], [10], [12]-[13]. Alternatively, non-contact sensors, i.e. infrared (IR), can be used [7]-[8]; however, they are relatively expensive and require visual access to the magnet surface, which is feasible in many surface PMSM (SPMSM) designs but not in most interior PMSM (IPMSM) designs. For all the cases, the use of temperature sensors increases the system cost and can cause reliability concerns. Therefore, direct PM temperature measurement is usually not implemented in standard machines.

As an alternative to direct measurement, PM temperature can be estimated. Temperature estimation reported in the literature include thermal model based methods [3]-[6], back electromotive force (BEMF) based methods [15]-[21] and methods based on the injection of some form of test signal [7][13]. Thermal models require precise knowledge of the machine geometry, materials and cooling system for obtaining a precise model for each machine design [3]-[6]. The drift in thermal model parameters due to reduction in the cooling capability commonly caused by obstructed cooling flow are known to cause errors. This can cause thermal model based temperature monitoring to fail considering that reduced cooling is an important cause of abnormal temperature rise. BEMF based methods estimate the magnet temperature from the estimated PM flux linkage [15]-17]. They can provide precise estimates of temperature when the machine rotates above a certain speed, but are not viable when the machine operates at standstill or low speed. Furthermore, knowledge of the machine inductances is needed, which can significantly change with the operating condition of the machine [18]-[21]. Finally signal injection methods require injection of an additional high frequency signal superimposed on the fundamental excitation [7]-[13]. While these methods can operate at any speed, including zero speed, they can have some adverse effects on the operation of the machine (e.g. extra losses, noise and vibration).

Control of PMSMs requires rotor position measurement/ estimation, as well as the magnet polarity detection prior to startup of the machine. The rotor position of PMSMs is typically measured using speed/position sensors, e.g. encoders, resolvers... [28]-[31], while the magnet polarity is commonly measured using digital hall-effect sensors [22]-[26]. Consequently, commercial PMSMs are frequently equipped with both types of sensors [28]- [31]. A large variety of digital [22]-[26] and analog [28], [30], [32]-[34], [41]-[45] hall-effect 


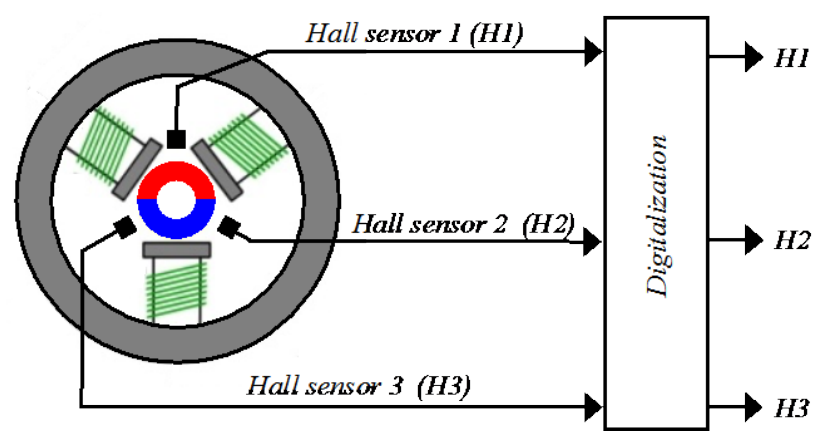

Fig. 1. Block diagram of hall-effect sensors and digitalization.

sensors are available for this purpose. The main idea of the new method proposed in this work is to estimate the temperature of the PM using the flux measurements obtained from low cost hall-effect sensors [22]-[31], [46]. For a majority of PMSM servo motor applications, it is possible to take advantage of hall sensors available in the motor for initial position estimation with minor modification to the hardware. The signal provided by the sensors can be used to measure the magnetic flux strength, from which the magnet temperature can be estimated. Experimental test results on 2 IPMSMs will be provided to show that the proposed method can provide noninvasive estimation of PM temperature for monitoring and protection against excessive temperature rise with minor modifications to existing PMSM drives, and without motor parameter information.

\section{HALl SENSOR-BASED ESTIMATION OF PM TEMPERATURE}

Control of PMSMs requires knowledge of the absolute position of the rotor, meaning that some type of absolute position sensor is required. However, the majority of the PMSMs used for servo motor control applications rely on incremental encoder measurements for obtaining the position [28]-[31]. Knowledge of the initial rotor position is required for a controlled start up of the motor, three hall-effect sensors are typically installed for this purpose [22]-[28]. The signal provided by these sensors is usually digitized, as only the presence of the magnet needs to be detected, as shown in Fig. 1 [28], [30], [34]. While digitization simplifies the signal acquisition and increases the noise immunity, it removes information contained in the analog signal, which could potentially be used to monitor the magnet condition, and more specifically the magnet temperature. Alternatively, hall-effect sensors providing an analog signal [32]-[34] combined with analog inputs can be used. Examples of commercial drives using the analog signal from the hall-effect sensors can be found in [34]-[35].

PM temperature can be estimated from the analog measurements of flux density obtained from the hall sensors. The relationship between the PM flux, $B$, and the temperature is given by

$$
\mathrm{B}\left(T_{P M}\right)=\mathrm{B}\left(T_{P M 0}\right) \cdot\left(1+\alpha_{P M} \cdot\left(T_{P M}-T_{P M 0}\right)\right),
$$

where $T_{P M}, T_{P M 0}$ are the present and reference PM temperatures, and $\alpha_{P M}$ is the per unit temperature coefficient of the PM

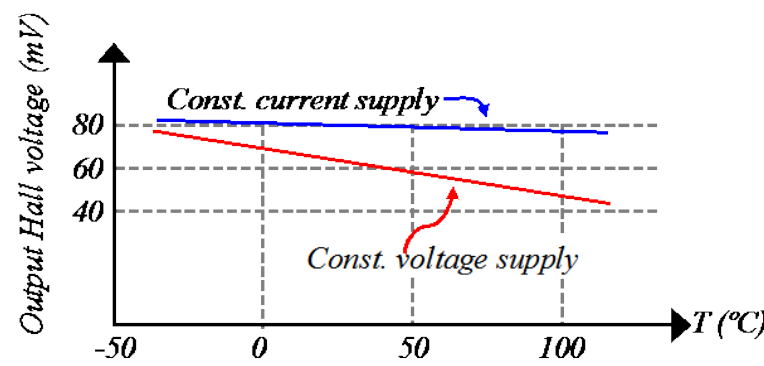

Fig. 2. Hall-effect sensor output voltage vs. temperature for the case of a constant voltage supply and a constant current supply when flux density (B) of $\approx 50 \mathrm{mT}$ is applied to the sensor.

material. The hall effect field sensor produces a voltage $V_{P M}$ $\left(T_{P M}\right)$ which is proportional to the field, the PM temperature can be then estimated from (2)

$$
\mathrm{T}_{P M}=\mathrm{T}_{P M 0}+\left(V_{P M}\left(T_{P M}\right) / V\left(T_{P M 0}\right)-1\right) / \alpha^{\prime},
$$

where $\alpha^{\prime}$ is coefficient $\alpha_{P M}$ that takes the temperature influence of hall sensor gain into account.

It can be seen from (2) that the PM temperature, $T_{P M}$, can be estimated from the analog hall effect sensor voltage output. While the principles of the proposed method are simple, there are several issues that need to be considered for its implementation, as they can influence the performance and reliability of $T_{P M}$ estimation. The potential issues to be considered can be summarized as:

- Interference of the field produced by the stator current. Location and angle of orientation of hall sensor(s) should be selected to have high sensitivity to the PM field while having low sensitivity to the field produced by the stator current. This issue is analyzed in detail in Section III. Compensation of the $T_{P M}$ estimate for stator currentproduced flux interference, and calibration of the $T_{P M}$ estimation algorithm is described in IV.

- Effect of the non-linear behavior of PM and hall sensor properties, i.e. $\alpha_{P M}$ and $\alpha$ ' are temperature-dependent. The variation in $\alpha_{P M}$ with temperature is expected to be between -0.1 to -0.11 for $T_{P M}$ below $125^{\circ} \mathrm{C}$, and between 0.1 to -0.15 for $T_{P M}$ below $175^{\circ} \mathrm{C}$ according to [11]. $\alpha_{P M}$ depends on the specific NdFeB material used, and can be obtained from the PM manufacturer to estimate $T_{P M}$ from the flux density measurements. Since the main objective of estimating $T_{P M}$ in this paper is for protection purposes, it is expected that the preciseness of the temperature estimate would be sufficient with the PM material properties provided in the manufacturer specs.

- Non-uniform temperature distribution within the PM. There will be some difference between the hottest and coolest part of the PM depending on the structure and how the motor is cooled. This issue is not taken into account in this work since the purpose is to monitor $T_{P M}$ for protection, where action could be taken when it is excessive. The sensor will provide a lumped measurement, which implicitly assumes that the PM temperature is uniform. Consequently, non-uniform temperature distribution cannot 


\begin{tabular}{|l|l|}
\hline \multicolumn{2}{|c|}{ Table I. Machine parameters } \\
\hline$P_{\text {RATED }}(\mathrm{kW})$ & 7.5 \\
\hline$I_{\text {RATED }}(\mathrm{A})$ & 14 \\
\hline$\omega_{\text {RATED }}(\mathrm{rpm})$ & 1800 \\
\hline Stator slots & 36 \\
\hline Poles & 6 \\
\hline Rotor radius (mm) & 53.4 \\
\hline Magnets & $\mathrm{N} 42 \mathrm{SH}$ \\
\hline Magnet dimensions: width, height and length (mm) & $42 \times 6 \times 10$ \\
\hline Magnet position from shaft center (mm) & 45.6 \\
\hline Airgap length (mm) & 0.8 \\
\hline Inner stator radius (mm) & 54.2 \\
\hline Outer stator radius (mm) & 84 \\
\hline
\end{tabular}

a)

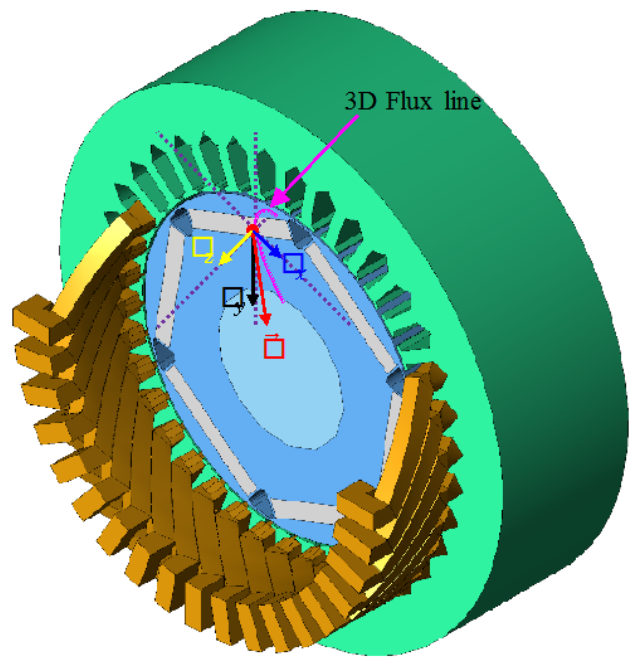

Flux measurement

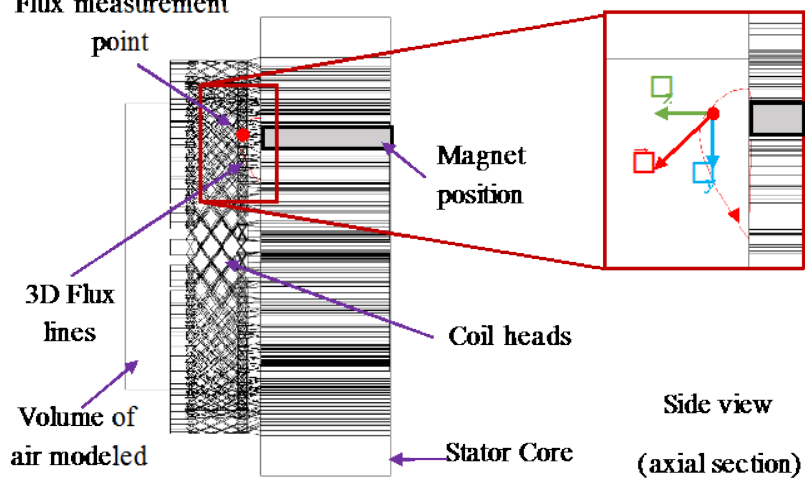

b)

Fig 3. Schematic representation of the machine, including hall effect sens or location and coordinate system for the field vector. Part of the end win dings has been removed intentionally from Fig. 3a to show the coordinate system used for field vector.

be detected. It must be noted however that this limitation is common to all temperature estimation methods.

- Dependency of the hall-effect sensor output on the sensor temperature. This strongly depends on the type of sensor and on the circuit used to feed it. Hall-effect sensors are commonly fed from a constant voltage source [28]-[31]. There is a significant dependency of the sensor output voltage on its temperature in this case (see Fig. 2), which could be a concern for the use of the sensor for magnet

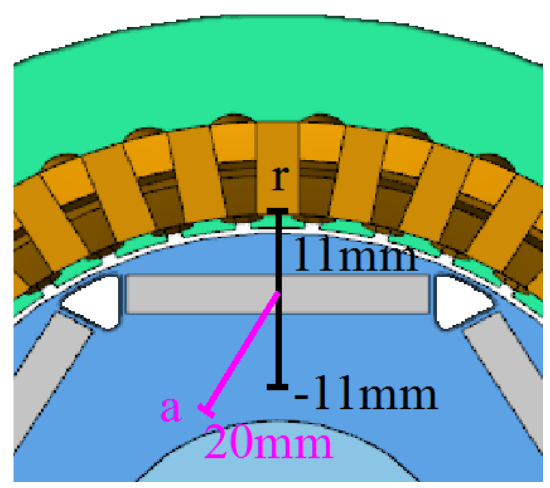

Fig 4. Coordinate system for the field sensor displacement in radial (r) an d axial (a) directions for model shown in Fig 3.

temperature estimation. However, temperature compensated hall-effect sensors can be easily found [33], [36]-[40]. Alternatively, the hall-effect sensors can be fed from a constant current source. The sensitivity of the sensor to temperature practically being dramatically reduced, as shown in Fig, 2. .

\section{FINITE ELEMENT ANALYSIS (FEA)}

As mentioned in Section II, sensor location should be chosen to have high sensitivity to the PM field and low sensitivity to the field produced by the stator current. While this concern also exists when the hall effect sensors are used for initial rotor position estimation [22]-[27], the requirements for temperature estimation are more demanding, as not only the presence of the magnet but a precise measurement of its strength is required. A 3 dimensional finite element (FE) model, see Fig. 3 and 4, was used to analyze this issue. A schematic representation of the PMSM including the initial location of the sensor and the xyz coordinate system used for the analysis is shown in Fig. 3. A more detailed representation is shown in Fig. 4. The sensor provides field measurement in the $x y z$ coordinates, which is useful for determining its optimal orientation angle. The details of the ratings and dimensions of the simulated machine are summarized in Table I.

The measurements provided by the sensor in the $x, y$ and $z$ directions, when the sensor is displaced from the origin in the radial and axial directions (" $r$ " and "a” in Fig. 4 respectively) with no current in the stator windings (i.e $I_{d q}=0 \mathrm{~A}$ ), are shown in Fig. 5. It can be observed from Fig. 5a that the measured field in the $y$ and $z$ directions significantly changes when the sensor is displaced radially. The highest sensitivity occurs in the $z$ direction when the sensor is displaced $8 \mathrm{~mm}$ in radial direction. It is important to note however that in this case the sensor will be closer to the stator, which could result in an increased sensitivity to the field produced by the stator current, which is an undesirable feature. Finally, it can be seen from Fig. 5b that the measured fields in all the three directions decrease when the sensor is displaced in the axial direction, i.e. the sensor is moved further away from the magnet, as expected. 


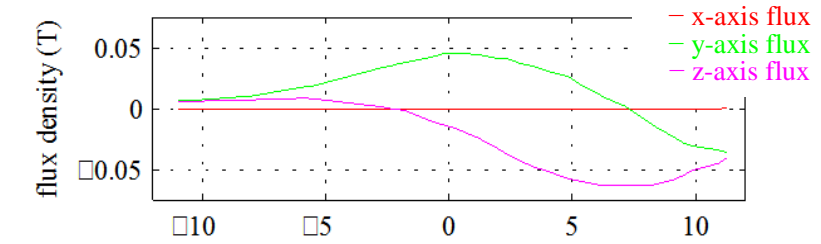

a)

radial displacement $(\mathrm{mm})$

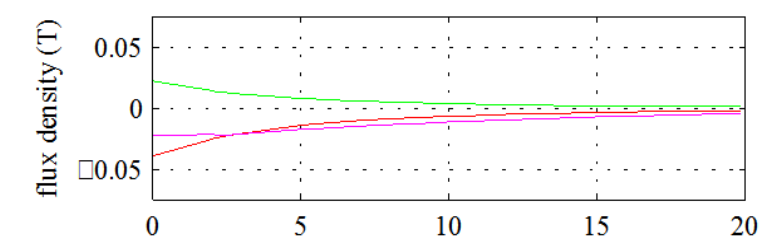

b)

axial displacement ( $\mathrm{mm})$

Fig. 5. FEA simulation results. Flux density measurement as a function o $\mathrm{f}$ the sensor displacement in the radial, a), and axial, b), directions. $\omega_{r}=2 *$ $\pi * 50 \mathrm{rad} / \mathrm{s}(1 \mathrm{pu}), I_{d q}=0 \mathrm{~A}$.

a)
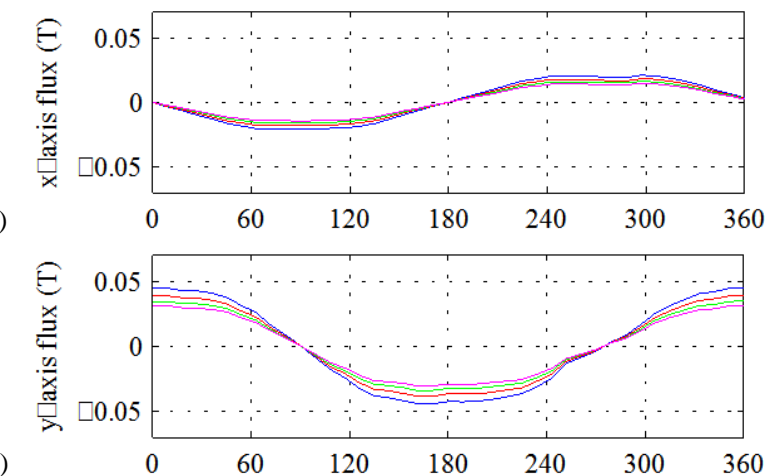

b)

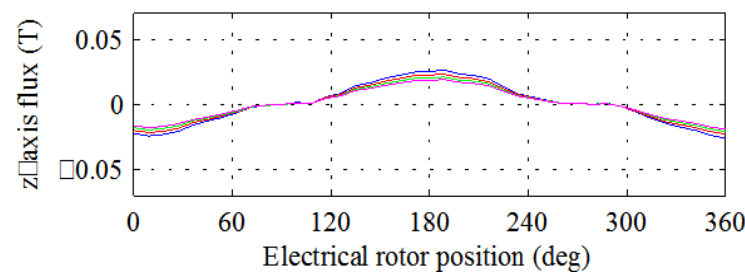

Fig. 6. FEA simulation results. Flux density variation due to magnet tem perature. Sensor position: $\mathrm{x}=0 \mathrm{~mm}, \mathrm{y}=0 \mathrm{~mm}, \mathrm{z}=5 \mathrm{~mm} . \omega_{r}=2 * \pi * 50 \mathrm{rad} / \mathrm{s}$ (1 pu), $I_{d q}=0$ A.

The field measured by the sensor as a function of the rotor angle, for different temperatures and when there is no fundamental current and the machine is rotating, is shown in Fig. 6. It can be observed that the measurements in all the three directions are sensitive to temperature variations and could therefore be used for temperature estimation; the sensitivity being highest in the $y$ direction (Fig. 6b).

The same field measurements as Fig. 6 but for different magnitudes of the $d$-axis current are shown in Fig. 7. The magnet temperature is kept constant in these simulation results. It can be observed that highest (undesired) sensitivity to the stator current occurs in the $y$ axis (Fig 7b), while the lowest (desired) sensitivity occurs on the $z$ axis (Fig 7b). The same field measurements as Fig. 7 but for different magnitudes of $q$ axis current are shown in Fig. 8. It can be observed in this case a)

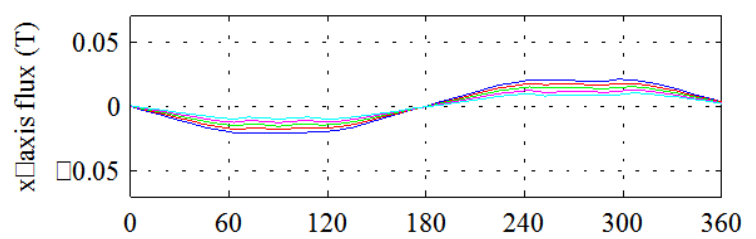

b)
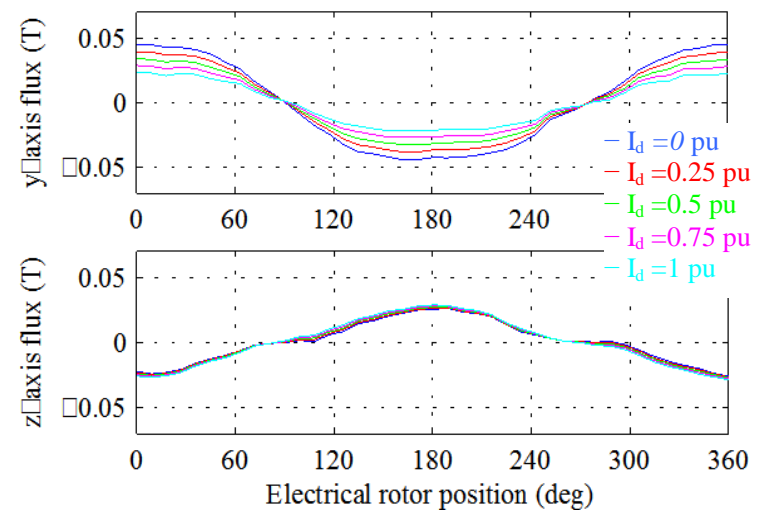

Fig. 7. FEA simulation results. Flux density variation due $I_{d}$ current injectio n. Sensor position: $\mathrm{x}=0 \mathrm{~mm}, \mathrm{y}=0 \mathrm{~mm}, \mathrm{z}=5 \mathrm{~mm}$. $\omega_{\mathrm{r}}=2^{*} \pi^{*} 50 \mathrm{rad} / \mathrm{s}(1 \mathrm{pu}), I_{q}=0$ A and magnet temperature $=20^{\circ} \mathrm{C}$.

a)

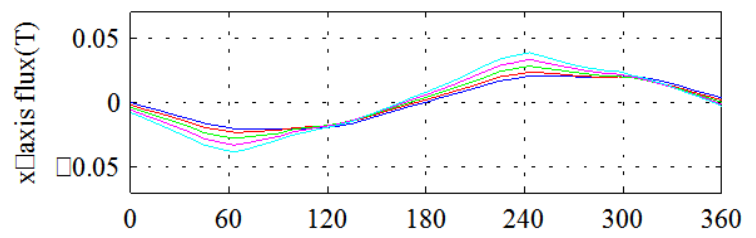

b)
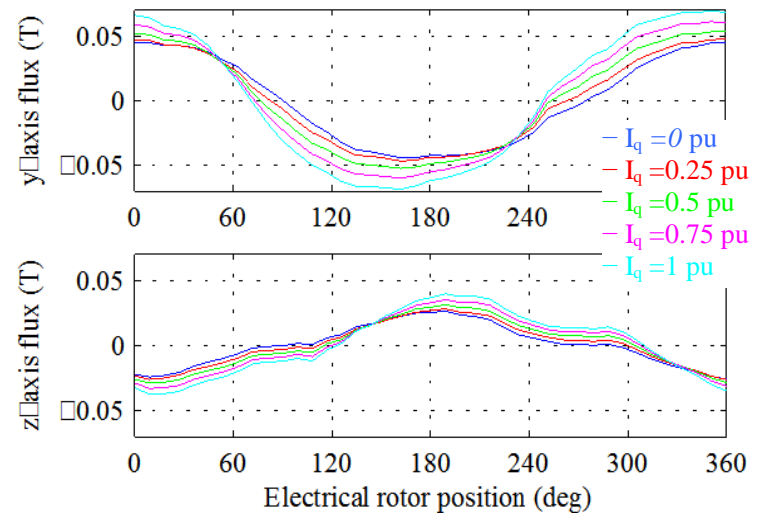

c)

Fig. 8.- FEA Simulation results. Flux density variation due $I_{q}$ current injectio n. Sensor position: $\mathrm{x}=0 \mathrm{~mm}, \mathrm{y}=0 \mathrm{~mm}, \mathrm{z}=5 \mathrm{~mm} . \omega_{r}=2^{*} \pi^{*} 50 \mathrm{rad} / \mathrm{s}(1 \mathrm{pu}), I_{d}=0$ A and magnet temperature $=20^{\circ} \mathrm{C}$.

that the measurements are sensitive to the field produced by the q-axis current in all the axes $(x, y$, and $z$ ), with the highest (undesired) sensitivity for the $y$-axis and the lowest (desired) sensitivity for the $z$-axis. It can be concluded from Figs. 6-8 that aligning the field sensor with the $z$ direction would provide the best trade-off between the highest possible sensitivity to the PM field variation and the lowest possible sensitivity to the field produced by the stator $d$ and $q$-axes currents.

Finally, Fig. 9 shows the field measured by the sensors vs. time for a constant temperature and for different machine 
a)
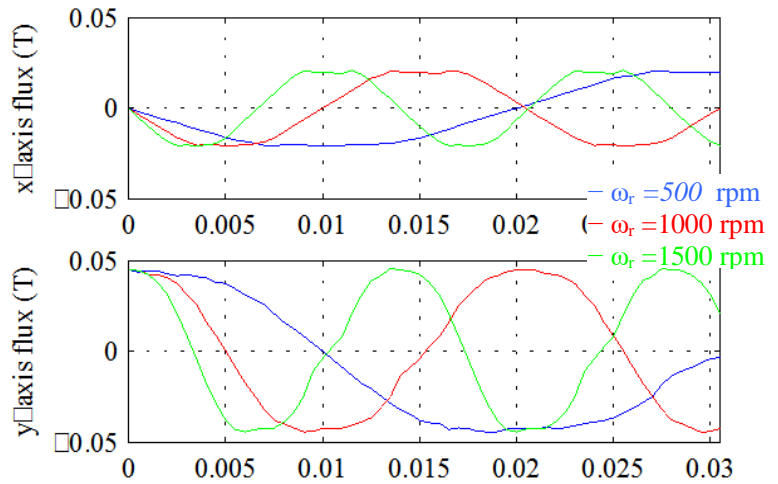

b)

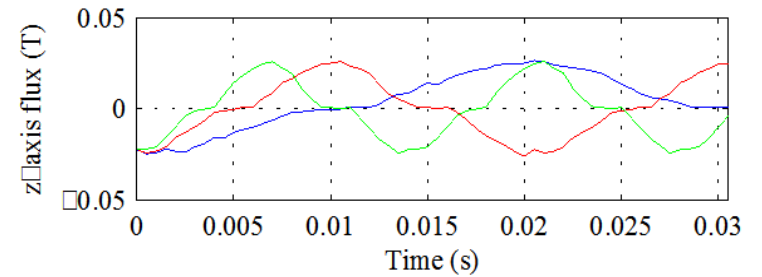

Fig. 9. FEA simulation results. Flux density variation with machine spee d. Sensor position: $\mathrm{x}=0 \mathrm{~mm}, \mathrm{y}=0 \mathrm{~mm}, \mathrm{z}=5 \mathrm{~mm}$. $I_{d q}=0$ A and magnet tempe rature $=20^{\circ} \mathrm{C}$.

speeds, when there is no fundamental current. It is observed from this figure that the sensors measurements are not affected by the rotor speed, which was an expected result as the magnet strength is a property of the magnet material, which do not depend on the speed.

\section{IMPLEMENTATION OF THE METHOD}

A commercial IPMSM (motor 1) equipped with standard field sensors (i.e. 1D sensors [33]) will be used to analyze practical implementation issues. Rated parameters are shown in Table II. The end shield of the non-drive end (NDE) was replaced with a custom designed and machined shield for mounting hall sensors A and B, an infrared (IR) thermal sensor, and a position encoder, as shown in Figs. 10 and 11. Two hall sensors, A and B, were installed for measurement of the flux density, as described in Section III. The hall sensors were oriented in the axial direction, i.e. z-axis direction (see Fig. 1), which is expected (based on FE analysis) to provide high sensitivity to PM flux and low sensitivity to stator current. The hall sensors were fixed inside the motor to the end of the extension rods that protrude into the end shield, as shown in Fig. 11a. The rods were designed to be adjustable from the outside in the axial (z-axis) and radial (y-axis) directions by \pm 5 $\mathrm{mm}$ to be able to perform testing with halls sensors at different positions, as shown in Fig. 11b. Once the position of the hall sensors was determined, they were fixed by applying gypsum to the rod and sensor internal and external to the end shield, as shown in Fig. 10a.

A commercial IR thermal sensor was also fixed at a position in the end shield to point to the radial center of the PM of the rotor shown in Fig. 11b. It measures the average temperature of an area of a $5 \mathrm{~mm}$ diameter circle (from the 10 $\mathrm{cm}$ distance from the sensor) with a sampling frequency of $4.17 \mathrm{~Hz}$. It should be noted that the measurement area sweeps

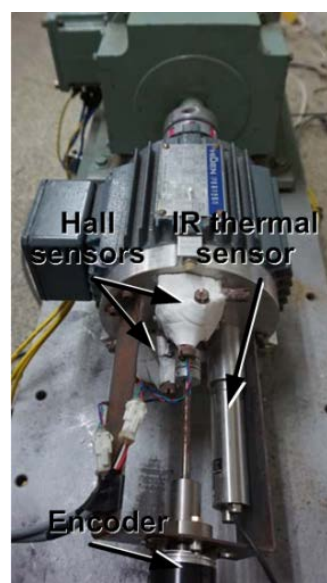

a)

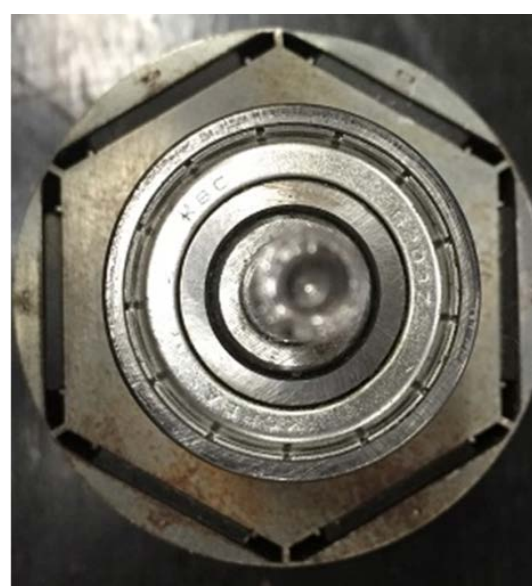

b)
Fig. 10.- Motor 1 experimental setup: external view of (a) motor and NDE shield with hall sensors, IR thermal sensor, and encoder; (b) rotor surface.

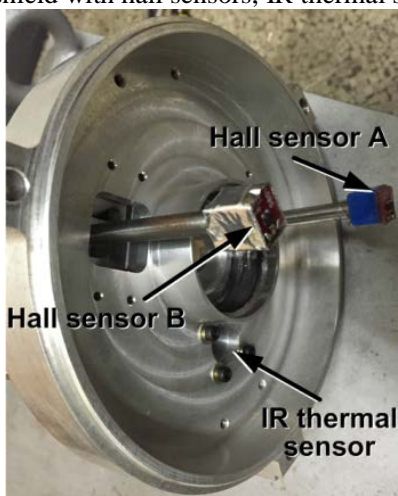

a)

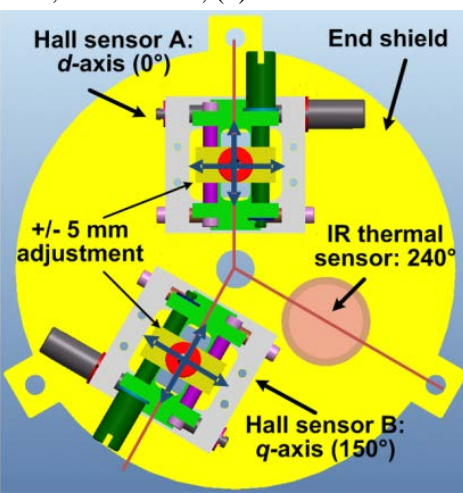

b)
Fig. 11.-Motor 1 experimental setup: (a) internal view of NDE shield with hall sensors A and B, and IR thermal sensor; (b) design drawing of NDE shield with adjustable hall sensor position.

\begin{tabular}{|l|l|}
\hline \multicolumn{2}{|c|}{ Table II. Motor 1 parameters } \\
\hline$P_{\text {RATED }}(\mathrm{kW})$ & 2.2 \\
\hline$I_{\text {RATED }}(\mathrm{A})$ & 8.2 \\
\hline$V_{\text {RATED }}(\mathrm{V})$ & 212 \\
\hline Poles & 6 \\
\hline
\end{tabular}

both PM and the core as the rotor rotates; therefore, the average temperature of the PM axial surface and rotor core is being measured. Although this results in a not fully accurate measurement of $T_{P M}$, it is sufficient to show the trend in the change in the PM temperature. To increase the rate of temperature rise, the shaft-cooling fan was removed and the motor was covered with thermal insulation.

A TMS320F28335 $\mu$-controller was used to implement the IPMSM control, as well as acquisition of the temperature and hall sensor data. Considering the large PM thermal time constant, $T_{P M}$ estimate was calculated once every minute from the rms value of the flux. The test motor was mechanically coupled to an $1800 \mathrm{rpm}$ DC machine operated as a generator feeding a resistor bank (constant field voltage) to load the IPMSM test motor, as shown in Fig. 10a. 


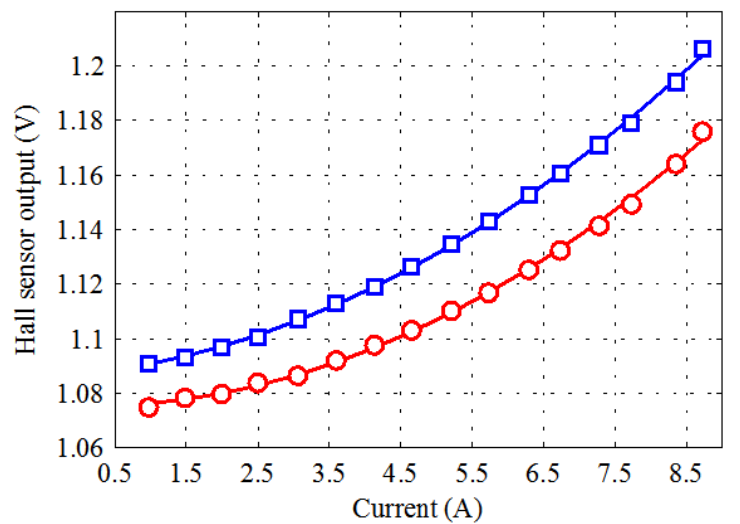

Fig. 12. rms values of hall sensor A ( $\square$ ) and B (o) output voltage measurements as a function of the injected stator current between $1 \mathrm{~A}$ and 8.7 A with $T_{P M}$ at room temperature, and curve-fitted quadratic curve used for compensation for stator current interference (Motor 1).

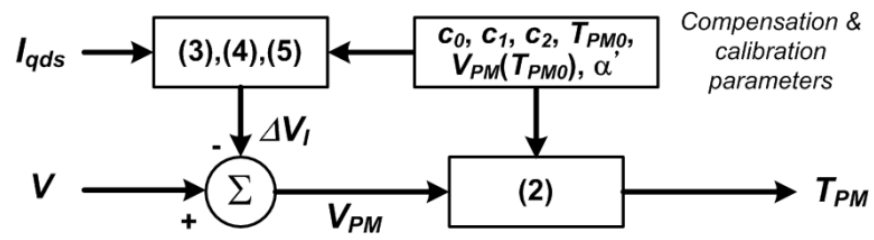

Fig. 13. Flowchart of proposed calibration and compensation algorithm for hall sensor based PM temperature estimation.

\section{A. Compensation for the Influence of Stator Current Interference}

The PM leakage flux is measured in the z-axis direction (Fig. 1). It was concluded from Fig. 7-8 that the hall sensor measurement is affected either by the $\mathrm{d}$ and q-axes currents. Therefore, the influence of the stator winding current on the hall sensor measurement must be compensated to accurately estimate the PM temperature. A straightforward approach for compensating for the stator current produced flux is to measure the hall sensor output as a function of the stator current magnitude at a fixed PM temperature during a commissioning process. This data can be stored and further used for real-time compensation during normal operation of the drive.

The rms output voltage from the two hall sensors, A and B, shown in Fig. 11 (measuring the z-axis direction flux) are shown in Fig. 12. Data was obtained with the stator current magnitude changing between 1 and $8.7 \mathrm{~A}$ in discrete steps (rated current is $8.2 \mathrm{~A}$ ), while maintaining the PM under thermal equilibrium at room temperature. The angle between the $d$ - and $q$-axis currents was maintained such that maximum torque per ampere (MTPA) is achieved when operated in the constant flux region at or below rated speed. As expected from the FEA simulation results, the hall sensor measurements are influenced by the stator current magnitude, following a quadratic variation with the stator current. It can also be seen that the measurements from both hall sensors are slightly different, meaning that, as expected, the measurement is sensitive to the sensor location. The rate of change due to the stator current is also different for the two hall sensors as their position relative to the PM has not been precisely adjusted.
Tests at different speeds were conducted; they are not shown due to space constraints. It was concluded from those testes that the degree of interference is independent of the rotor speed, which is consistent with the simulation results shown in Fig. 9.

The hall sensor rms output voltage as a function of stator current, $V_{I}$, can be calculated from the stator current rms value, $I_{q d s}$, by fitting the data points with a quadratic curve (3).

$$
\mathrm{V}_{I}\left(I_{q d s}\right)=c_{2} I_{q d s}^{2}+c_{1} I_{q d s}+c_{0},
$$

where $c_{0}, c_{1}$, and $c_{2}$ are coefficients that can be determined to minimize the error between the calculated and measured $V_{I}$. The fitted curve of $V_{I}$ plotted with $c_{0}, c_{1}$, and $c_{2}$ values obtained using a commercial software tool is also shown in Fig. 12. The "change" in $V_{I}, \Delta V_{I}$, can be calculated from (4).

$$
\Delta \mathrm{V}_{I}\left(I_{q d s}\right)=\mathrm{V}_{I}\left(I_{q d s}\right)-\mathrm{V}_{I}(0)=\mathrm{V}_{I}\left(I_{q d s}\right)-c_{0} \text {. }
$$

$\Delta V_{I}$ can be decoupled from the hall sensor voltage measurement, $V$, for obtaining the compensated hall sensor measurement (5), $V_{P M}$, to estimate the PM temperature from (2).

$$
\mathrm{V}_{P M}=V-\Delta \mathrm{V}_{I}\left(I_{q d s}\right) .
$$

With the compensation for the interference from the flux produced by the stator current, $V_{P M}$ represents the hall sensor measurement of PM flux. The coefficients for the quadratic equation $\Delta V_{I}\left(I_{q d s}\right)$ are required to be obtained once for the motor for the fixed location of the hall sensor. It is finally noted that the calibration/compensation strategy shown in Fig. 13 and described by (3)-(5) can be applied when the $d$ - and $q$ axis currents are controlled using a strategy different from MTPA.

\section{B. Calibration of Hall Sensor}

It can be seen in Fig. 12 that the hall sensor measurement is sensitive to the location of the sensor. There also could be variance in the PM location within the rotor and in the hall sensor characteristics from motor to motor that can influence the accuracy of the $T_{P M}$ estimate. Therefore, a calibration procedure for the individual motors is desirable for improving the quality of the $T_{P M}$ estimate. Calibration can be performed during the quality assurance testing stage for the individual motors after the motor has been assembled. It is also possible to implement the calibration procedure in the inverter and automate it as a built-in feature.

The uncompensated hall sensor measurement $V\left(T_{P M 0}\right)$ at a known reference temperature, $T_{P M O}$, can be obtained when motor (and PM) is at thermal equilibrium at which $T_{P M 0}$ is identical to the known ambient temperature. The $V_{I}$ curve as a function of $I_{q d s}$ can be easily obtained by measuring $V_{I}$ while controlling $I_{q d s}$ at different values for a short period of time. The data can be obtained at any speed since $V_{I}\left(I_{q d s}\right)$ is independent of rotor speed (see Fig. 9). Coefficients $c_{0}, c_{1}$, and $c_{2}$ can be calculated from the $V_{I}\left(I_{q d s}\right)$ curve and stored to perform the compensation algorithm shown in (3)-(5) to obtain $V_{P M}$ and $T_{P M}$ while the motor is operating. The compensated hall sensor measurement $V_{P M}\left(T_{P M 0}\right)$ can also be calculated and stored. The flowchart of the proposed algorithm with the 
a)

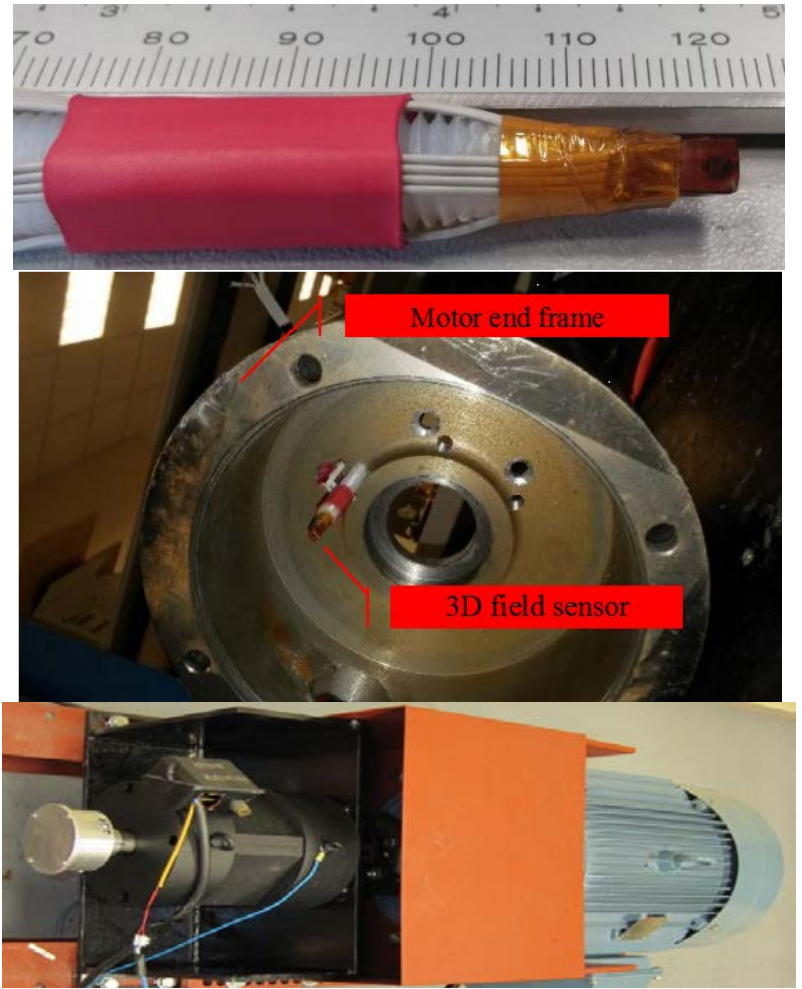

Fig. 14.- Motor 2: a) 3D field sensor, b) field sensor mounting and c) experim ental setup.

compensation and calibration procedures is summarized in Fig. 13.

\section{EXPERIMENTAL RESULTS}

Experimental results using a $3 \mathrm{D}$ field sensor prototype to verify the FEM results presented in section III and experimental results using standard, i.e. commercial, 1D field sensors will be shown in this section.

\section{A. Experimental results using a $3 D$ field sensor prototype}

The field sensor prototype that will be used for the experimental verification for test motor 2 is shown in Fig. 14(a) [32]. Three field sensors are mounted to measure field in $\mathrm{x}, \mathrm{y}$ and z-axis directions. The field sensor is an ion-implanted magnetic field sensor made of mono-crystal gallium arsenide (GaAs) semiconductor that provides a wide measuring range 0 3T [32]. Fig. 14(b) shows the field sensor mounting, a hole has been practiced in the end frame of the machine to place the 3D field sensor. The test bench used for the experimental verification on test motor 2 is shown in Fig. 14(c). Parameters and design of the PMSM are the same as the machine used for simulation shown in Table I, Figs. 3 and 4.

The flux measured along y-axis direction for a machine temperature of $\approx 25^{\circ} \mathrm{C}$ and a speed of $\approx 2 \pi 50 \mathrm{rad} / \mathrm{s}$, with no fundamental current $\left(I_{d q}=0 \mathrm{~A}\right)$, is shown in Fig. 15. The FFT of the flux measurement of Fig. 15 is shown in Fig. 16. The magnitude of the fundamental component of the flux density (component at $\approx 50 \mathrm{~Hz}$, see Fig. 16), will be used to experimentally evaluate the variation of the magnet flux with

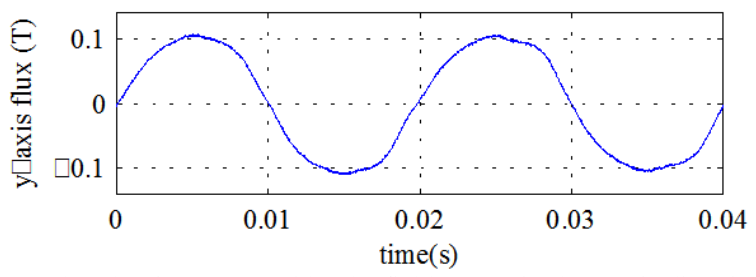

Fig. 15.- Flux density measured by the field sensor in the y axis direction vs. ti me. Sensor position: $\mathrm{x}=0 \mathrm{~mm}, \mathrm{y}=0 \mathrm{~mm}, \mathrm{z}=4.5 \mathrm{~mm} . I_{d q}=0 \mathrm{pu}, \omega_{r}=2 * \pi * 50 \mathrm{rad} / \mathrm{s}$ a nd magnet temperature $\approx 25^{\circ} \mathrm{C}$ (Motor 2).

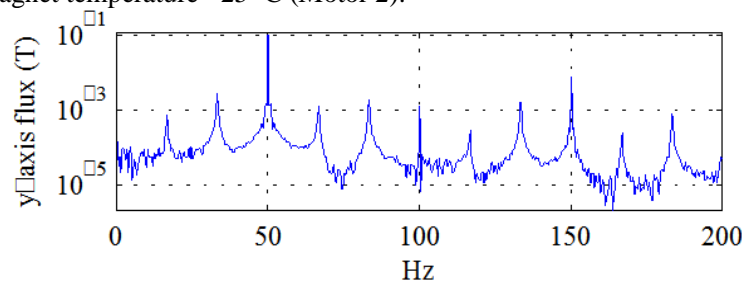

Fig. 16.- FFT of the flux density in Fig. 15.

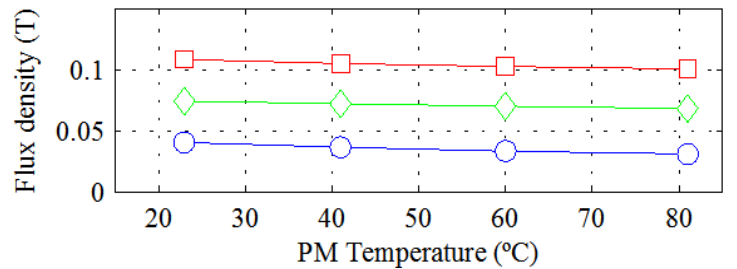

Fig. 17.- Flux density measured with the field sensor in x (o), y ( $\square$ ) and z ( $\diamond)$ a xis directions (see Fig. 3) vs. the magnet temperature. Sensor position: $x=0 \mathrm{~mm}$, $\mathrm{y}=0 \mathrm{~mm}, \mathrm{z}=4.5 \mathrm{~mm} . I_{d q}=0 \mathrm{pu}, \omega_{r}=2 * \pi * 50 \mathrm{rad} / \mathrm{s}$ (Motor 2 )

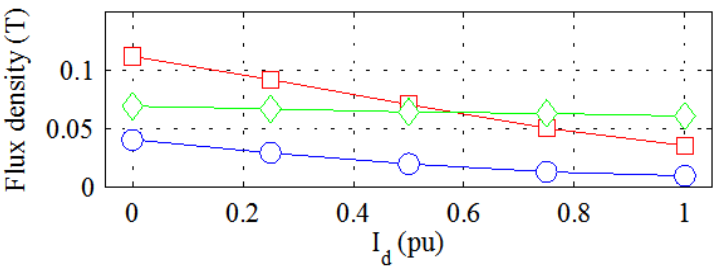

a)

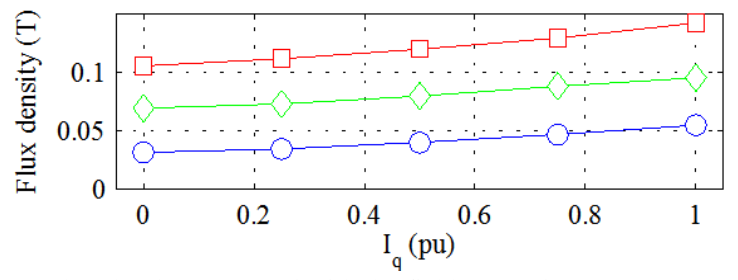

Fig. 18.- Flux density measured with the field sensor in $\mathrm{x}(\mathrm{o}), \mathrm{y}(\square)$ and $\mathrm{z}(\diamond) \mathrm{a}$ xis directions (see Fig. 3) vs. $I_{d}, a$ ), and $I_{q}, b$ ). Sensor position: $x=0 m m, y=0 m$ $\mathrm{m}, \mathrm{z}=4.5 \mathrm{~mm}$. $I_{d q}=0 \mathrm{pu}, \omega_{r}=2 * \pi * 50 \mathrm{rad} / \mathrm{s}$ (Motor 2).

temperature, as well as with $I_{d}$ and $I_{q}$ currents (see Fig. 17 and 18).

The magnitude of the fundamental components of the flux density measured using the field sensor along $\mathrm{x}, \mathrm{y}$ and $\mathrm{z}$-axis directions when the temperature of the machine changes from $\approx 25^{\circ} \mathrm{C}$ (i.e. room temperature) to $\approx 82^{\circ} \mathrm{C}$, is shown in Fig. 17 . The machine was first heated up to $\approx 82^{\circ} \mathrm{C}$ by injecting fundamental current and then it was cooled down naturally without injecting fundamental current $\left(I_{d q}=0 \mathrm{~A}\right)$. It is observed that the field measured along the three axis directions increases as the magnet temperature decreases, the variation of the measured field being similar in all the three directions. These 
a)

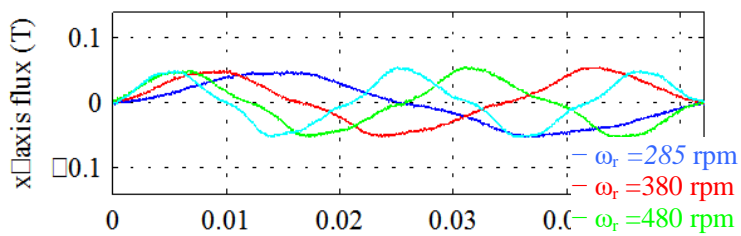

b)
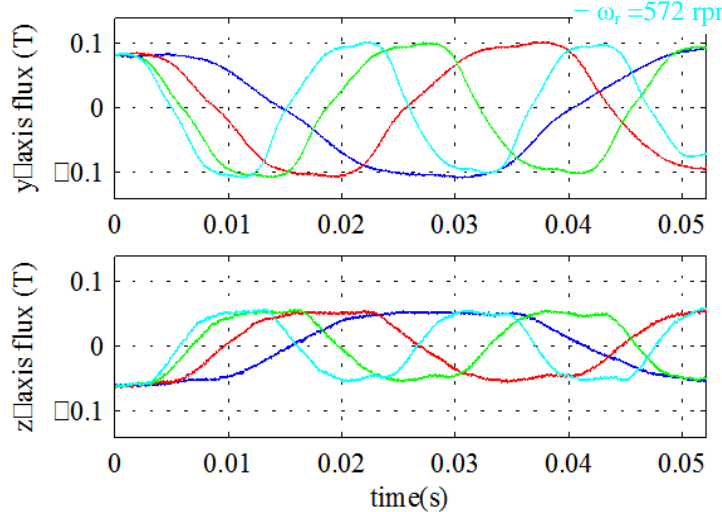

c)

Fig. 19. Experimental results. Flux density with speed. Sensor position: $\mathrm{x}=0 \mathrm{~mm}, \mathrm{y}=0 \mathrm{~mm}, \mathrm{z}=5 \mathrm{~mm} . I_{d q}=0 \mathrm{~A}$ and magnet temperature $\approx 25^{\circ} \mathrm{C}$.

results are in good agreement with the FEA results that are shown in Fig. 6.

The magnitude of the fundamental component of the flux density measurements using the 3D field sensor along $\mathrm{x}, \mathrm{y}$ and z-axis directions when the $d$ and $q$ axis currents change from 0 to $1 \mathrm{pu}$, are shown in Fig. 18. It can be clearly observed that the field along the $z$-axis direction shows the lowest sensitivity to the field produced by the stator $d$-axis current, which is in good agreement with the simulation results shown in Fig. 5. It can also be observed from Fig. 18(b) that the sensitivity to the to the field produced by the stator $q$-axis current is very similar along $x, y$ and $z$-axis directions, meaning that there is no preferential direction, i.e. a direction with very small or even negligible sensitivity to the field produced by the stator $q$-axis current.

Finally, the fields measured with the 3D sensor along the $x$, $y$ and $z$-axis directions vs. time for different speeds, are shown in Fig. 19. The trends observed in these results are consistent with the simulation results shown in Fig. 9. However, it is observed that there are relative large differences in the flux density peaks provided by FEM (see Fig. 9) and experimentally measured (see Fig. 19). There are two major sources for these discrepancies: differences in the end winding shape between the FE model and the real machine resulting from manufacturing tolerances, and tolerances for the sensor assembling.

It can be concluded from the previous experimental results that $z$-axis direction provides the best trade-off between an increased sensitivity to the PM field and the decreased sensitivity to the field produced by the stator current, and that the measurements are independent of rotor speed.

\section{B. Experimental results using standard field sensors}

Further experimental results have been carried out on motor 1, i.e. commercial machine equipped with commercial field sensors [33], where the parameters of motor 1 are shown in Table II. To verify the proposed $T_{P M}$ estimation method under temperature variation, the load of the motor was varied by controlling the speed of the motor. The load of the machine is proportional to the square of the rotor speed with the field voltage and load resistor values fixed. The field voltage of the DC motor was adjusted to a constant value so that the motor draws stator current at its rated value of $8.2 \mathrm{~A}$ at $1800 \mathrm{rpm}$. The speed was controlled to be constant for 2 hrs. at 1800, 900, and $1350 \mathrm{rpm}$, respectively, for a total of $6 \mathrm{hrs}$. The stator current decreased from 8.2 A to $4.7 \mathrm{~A}$, and increased to $6.6 \mathrm{~A}$ when the speed was controlled at 900 and 1350 rpm, respectively.

The IR sensor measurements of $T_{P M}$ and the hall sensor A measurements before $(V)$ and after $\left(V_{P M}\right)$ compensation of stator current interference, are shown in Fig. 20a-b, respectively, for the $6 \mathrm{hr}$ interval. The contribution of the stator current to the hall sensor measurements $\left(\Delta V_{I}\right)$ for sensor A was subtracted from the rms value of the raw measurements (V) using (3)-(5) to compensate for the interference (Fig. 10) to obtain $V_{P M}$, as described in section IV. It can be seen in Fig. $20 \mathrm{~b}$ that the dc offset of the hall sensor output voltage level depends on the stator current level. There is no shift in the dc offset level for the compensated $V_{P M}$, and it decreases with increase in magnet temperature, and vice versa, as expected. The $T_{P M}$ estimate shown in Fig. 20c was calculated using (2) from $V_{P M}$. The value of $\alpha_{P M}$ for the $\mathrm{NdFeB}$ magnet was not available, and a constant value of $-1.2 \% /{ }^{\circ} \mathrm{C}$ was determined experimentally (an ideal hall sensor was assumed $\alpha^{\prime}=$ $\left.1.2 \% /{ }^{\circ} \mathrm{C}\right)$. The results show that the estimated $T_{P M}$ tracks the actual value with sufficient accuracy for thermal protection purposes (see Fig. 20d). An error between estimated and measured $T_{P M}$ as well as a delay can be observed (see Fig. 20a, 20c and 20d). Potential sources for the error and the delay are:

- IR thermal sensor $T_{P M}$ measurement is the average of the PM axial surface and rotor core temperatures, and therefore, is expected to be lower than the average PM temperature.

- The temperature distribution in the PM is non-uniform, where the temperature is highest in the center, and lowest at the point of measurement.

- Hall sensor flux density measurement represents the overall change in the PM flux, whereas the IR sensor $T_{P M}$ measurement represents the local temperature at the PM axial surface (coolest spot).

- Stator core magnetic characteristics (and therefore the characteristics of PM flux path) change with temperature (thermal time constant of stator core is much lower than that of the PM)

- Variation in $\alpha_{P M}$ and $\alpha$ with temperature is ignored (a constant value of $\alpha^{\prime}=-1.2 \% /{ }^{\circ} \mathrm{C}$ is used)

Regardless of these errors, the accuracy is sufficient for monitoring and detecting excessive temperature rise that can 


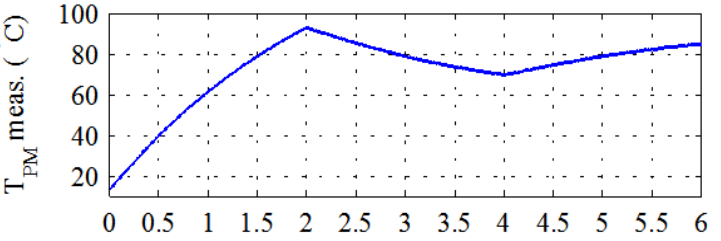

a)

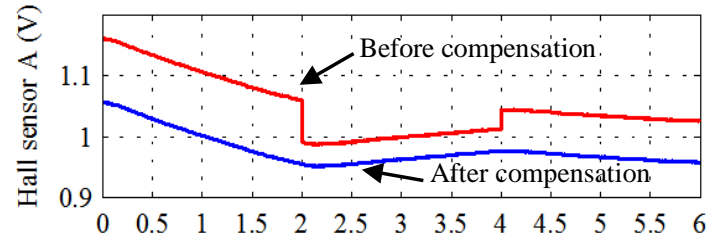

b)

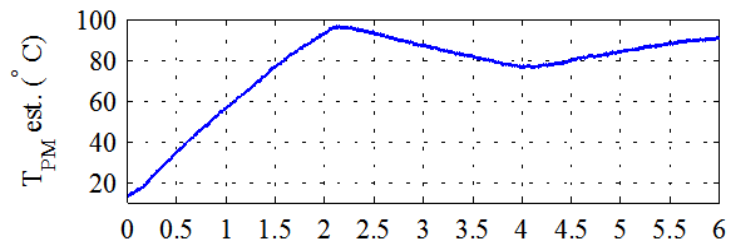

C)

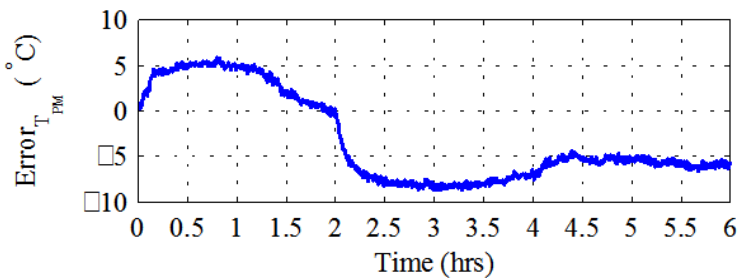

Fig. 20.- Experimental results (Motor 2): (a) IR thermal sensor measurement $\left(T_{P M}\right)$; (b) hall sensor A flux measurement before $(V)$ and after compensation $\left(V_{P M}\right)$; (c) $T_{P M}$ estimate from the hall sensor A; (d) $T_{P M}$ estimation error (Motor 1).

result in degradation or damage of the PM. An important advantage of the proposed method over conventional thermal model based thermal monitoring methods is that it is not influenced by changes in the cooling ability of the motor since flux is a direct indicator of PM temperature.

\section{CONCLUSION AND RECCOMENDATIONS FOR FUTURE WORK}

The feasibility of using the analog axial flux measurement obtained from hall sensors for estimating the temperature of the PM in PMSM has been evaluated in this paper. The proposed approach takes advantage of hall sensors used in most PMSM drives for detecting the initial rotor position. The method can provide non-intrusive estimation of the PM temperature with minimal design modifications to most existing systems. In addition, it is not influenced by changes in the thermal characteristics caused e.g. by reduced cooling ability, and does not require prior knowledge of motor parameters. FE and experimental results showed that measurement of the axial direction flux provides relatively high sensitivity to the PM flux with relatively low sensitivity to the stator current induced flux. Compensation of the effect of stator current is still needed; a compensation technique has been proposed and experimentally confirmed. An experimental study performed on 2 IPMSM systems showed that the PM temperature can be estimated with accuracy sufficient for thermal monitoring purposes.
In addition to PM temperature estimation discussed in this paper, other potential uses of analog hall effect sensors which are the subject of current research include

- PMSM fault detection: PM demagnetization (uniform or non-uniform), airgap eccentricities or stator core and winding insulation failures.

- High-resolution speed/position estimation.

\section{REFERENCES}

[1] P. Milanfar, and J.H. Lang, "Monitoring the thermal condition of permanentmagnet synchronous motors," IEEE Trans. on Aerosp. and Electron. Syst., vol. 32, no. 4, pp. 1421-1429, Oct. 1996.

[2] N. Bianchi, and T.M. Jahns "Design, analysis, and control of interior PM synchronous machines," Tutorial Course Notes, IEEE-IAS, Oct. 2004.

[3] A.M. EL-Refai, N.C. Harris, T.M. Jahns, and K.M. Rahman, "Thermal Analysis of Multibarrier Interior PM Synchronous Machine Using Lumped Parameter Model,” IEEE Trans. on Energy Convers., vol. 14, no.4, pp.303-309, June 2004.

[4] C. Kral, A. Haumer, and S.B. Lee, "A Practical Thermal Model for the Estimation of Permanent Magnet and Stator Winding Temperatures," IEEE Trans. on Power Electron., vol. 29, no. 1, pp. 455-464, Jan. 2014.

[5] B.H. Lee , K.S. Kim , J.W. Jung , J.P. Hong, and Y.K. Kim, "Temperature Estimation of IPMSM Using Thermal Equivalent Circuit," IEEE Trans. on Magn., vol. 48, no. 11, pp. 2949-2952, Nov. 2012.

[6] J. Grobler, S.R. Holm, and G. van Schoor, "Thermal Modeling of a High Speed Permanent Magnet Synchronous Machine,” Proc. of IEEE-IEMD, pp. 319-324, May. 2013.

[7] D. Reigosa, F. Briz, P. García, J. M. Guerrero, and M. W. Degner, "Magnet Temperature Estimation in Surface PM Machines Using High Frequency Signal Injection,” IEEE Trans. on Ind. Appl., vol. 46, no. 4, pp. 1468-1475, July/Aug. 2010.

[8] D. Reigosa, F. Briz, M. W. Degner, P. García, and J. M. Guerrero, "Magnet Temperature Estimation in Surface PM Machines During SixStep Operation,” IEEE Trans. on Ind. Appl., vol. 48, no. 6, pp. 23532361, Nov./Dec. 2012.

[9] D. Reigosa, D. Fernandez, H. Yoshida, T. Kato, and F. Briz "Permanent magnet temperature estimation in PMSMs using pulsating high frequency current injection,” IEEE Trans. on Ind. Appl., vol. 51, no. 4, pp. 3159-3168, July/Aug. 2015.

[10] D. Reigosa, D. Fernandez, T. Tanimoto, T. Kato, and F. Briz "Wireless Permanent Magnet Temperature \& Field Distribution Measurement System for IPMSMs,” Proc. of IEEE ECCE, pp 3996-4003, Sept. 2015.

[11] J.R. Hendershot, T.J.E. Miller, Design of brushless permanent magnet motors, Oxford Science Publications, USA, 1994.

[12] M Ganchev, C. Kral, and T. Wolbank, "Compensation of Speed Dependency in Sensorless Rotor Temperature Estimation for Permanent-Magnet Synchronous Motors,” IEEE Trans. on Ind. Appl., vol. 49, no. 6, pp. 2487-2495, Nov./Dec. 2013.

[13] M Ganchev, C. Kral, H. Oberguggenberger, and T. Wolbank, "Sensorless Rotor Temperature Estimation of Permanent Magnet Synchronous Motor,” Proc. of IEEE IECON, pp. 2018-2023, Nov. 2011.

[14] J. Hong,S.B. Lee, C. Kral, and A. Haumer, "Detection and Classification of Rotor Demagnetization and Eccentricity Faults for PM Synchronous Motors," IEEE Trans. on Ind. Appl., vol. 48, no. 3, pp. 923-932, May/June 2012.

[15] A. Specht, and L. Backer, “Observer for the Rotor Temperature of IPMSM,” Proc. of EPEIPEMC, 2010.

[16] O. Wallscheid, T. Huber, W. Peters, and J. Böcker, "Real-Time Capable Methods to Determine the Magnet Temperature of Permanent Magnet Synchronous Motors - A Review,” Proc. of IEEE-IECON, pp. 811-818, Nov. 2014. 
[17] A. Specht, O. Wallscheid, and J. Bocker, "Determination of rotor temperature for an interior permanent magnet synchronous machine using a precise flux observer," Proc. of IEEE IPEC, pp. 1501-1507, May 2014.

[18] K. Liu, and Z. Q. Zhu "Online estimation of the rotor flux linkage and voltage-source inverter nonlinearity in permanent magnet synchronous machine drives," IEEE Trans. on Power Electron., vol. 29, no. 1, pp. 418427, Jan. 2014.

[19] K. Liu, and Z.Q. Zhu "Mechanical Parameter Estimation of Permanent Magnet Synchronous Machines with Aiding from Estimation of Rotor PM Flux Linkage,” IEEE Trans. on Ind. Appl., vol. 51, no. 4, pp. 3115-3125, July/Aug. 2015.

[20] S. Underwood, and I. Husain, "On-line parameter estimation and adaptive control of permanent magnet synchronous machines," IEEE Trans. Ind. Electron., vol. 57, no. 7 pp. 2435-2443, Jun. 2010.

[21] S. Ichikawa, M. Tomita, S. Doki, and S. Okuma, "Sensorless control of permanent-magnet synchronous motors using online parameter identification based on system identification theory,” IEEE Trans. Ind. Electron., vol. 53, no. 2, pp. 363-372, Apr. 2006.

[22] S. Morimoto, M. Sanada, and Y. Takeda, "High performance currentsensorless drive for PMSM and SynRM with only low resolution position sensor," IEEE Trans. Ind. Appl., vol. 39, no. 3, pp. 792-801, May/Jun. 2003.

[23] J. Bu, L. Xu, T. Sebastian, and B. Liu, "Near-Zero Speed Performance Enhancement of PM Synchronous Machines Assisted by Low Cost Hall Effect Sensors," Proc. of IEEE APEC 98, pp 68- 74, Feb. 1998.

[24] A. Lidozzi, L. Solero, F. Crescimbini and A.D. Napoli, "SVM PMSM Drive With Low Resolution Hall-Effect Sensors," IEEE Trans. on Power Electron., Vol. 22, No. 1, pp. 282-290, Jan. 2007.

[25] F. G. Capponi, G. De Donato, L. Del Ferraro, O. Honorati, M. C. Harke, Robert D. Lorenz, "AC Brushless Drive With Low-Resolution HallEffect Sensors for Surface-Mounted PM Machines," IEEE Trans. on Power Electron., Vol. 47, No. 2, pp. 526-535, March 2006.

[26] M.C. Harke, G. De Donato, F.G. Capponi, T.R. Tesch, R.D. Lorenz, "Implementation Issues and Performance Evaluation of Sinusoidal, Surface-Mounted PM Machine Drives With Hall-Effect Position Sensors and a Vector-Tracking Observer," IEEE Trans. on Ind. Appl., vol. 44, no. 1, pp. 161-169, Jan./Feb. 2008.

[27] T.D. Batzel and K.Y. Lee, "Commutation Torque Ripple Minimization for Permanent Magnet Synchronous Machines with Hall Effect Position Feedback,” IEEE Trans. on Energy Convers., vol. 13, no. 3, Sept. 1998.

[28] Maxonmotorusa.com, “maxon sensor - Key information,” Mar. 23, 2017. [Online]. Available: http://www.maxonmotorusa.com/

[29] Siemens.com, "Three-phase synchronous motors based on permanent magnet technology,” Catalog D 86.2 • 2007, Mar. 23, 2017. [Online]. Available: http://w3.siemens.com/mcms/mc-solutions/en/motors/

[30] Technosoft.com, "Drive solutions for motion control applications," Product Overview, Mar. 23, $2017 . \quad$ [Online]. Available: http://www.technosoftmotion.com/en/products
[31] abbmotion.com, "Inteligent Servo Drives and Inteligent Motors," MotiFelex e180 motion control, Mar. 23, 2017. [Online]. Available: http://www.abbmotion.com/products/servodrives/overview.asp

[32] ChenYang - Sensors and Measurements, "Selection Guide of Hall Effect Sensor Elements/ICs,” CYSJ106C, Mar. 23, 2017. [Online]. Available: http://www.hallsensors.de

[33] Allegro Micro Systems LLC, "Low Noise, Linear Hall Effect Sensor ICs with Analog Output,” A1324, A1325, and A1326, Mar. 23, 2017. [Online]. Available: http://www.allegromicro.com

[34] faulhaber.com, "Brushless Dc servomotors," Series 3268...BX4, Mar. 23, 2017. [Online]. Available: https://fmcc.faulhaber.com

[35] Allegro Micro Systems LLC, "Ratiometric, Linear Hall-Effect Sensor ICs for High-Temperature Operation,” Datasheert A3717 and A3718, Mar. 23, 2017. [Online]. Available: www.allegromicro.com

[36] ChenYang - Sensors and Measurements, "CYL840X Linear Hall Effect Sensor ICs,” Datasheert CYL840X, Mar. 23, 2017. [Online]. Available: http://www.hallsensors.de

[37] Analog.com, "Ratiometric, Linear Output Magnetic Field Sensor," Datasheert AD22151, Mar. 23, 2017. [Online]. Available: http://www.analog.com

[38] ttelectronics.com, "Hallogic Hall-Effect Sensors," Datasheert OHS3151U, Mar. 23, 2017. [Online]. Available: http://www.ttelectronics.com

[39] micronas.com, "Linear Hall-Effect Sensor IC," Datasheert HAL401, Mar. 23, 2017. [Online]. Available: https://www.micronas.com

[40] Melexis.com, "Programmable Linear Hall-Effect sensor," Datasheert MLX90215, Mar. 23, 2017. [Online]. Available: https://www.melexis.com

[41] Y. F. Shi, Z. Q. Zhu, and D. Howe, "EKF-based Hybrid Controller for Permanent Magnet Brushless Motors Combining Hall Sensors and a Flux-Observer-based Sensorless Technique,” IEEE-IEMDC, pp. 14661472, May 2005.

[42] A. Simpkins, and E. Todorov, "Position Estimation and Control of Compact BLDC Motors Based on Analog Linear Hall Effect Sensors," American Control Conference, pp. 1948-1955, July 2010.

[43] X. Song, J. Fang, and B. Han, "High-Precision Rotor Position Detection for High-Speed Surface PMSM Drive Based on Linear Hall-Effect Sensors,” IEEE Trans. Power Electron., vol. 31, no. 7, pp. 4720 -4731, July 2016.

[44] Y. Y. Lee, R.-H. Wu, and S.T. Xu, "Applications of Linear Hall-Effect Sensors on Angular Measurement," IEEE International Conference on Control Applications (CCA), pp. 479 -482, Sept. 2011.

[45] L. Xiao, Y. Yunyue, Z. Zhuo, "Study of the Linear Hall-Effect Sensors Mounting Position for PMLSM," IEEE Conference on Industrial Electronics and Applications, pp. 1175 - 1178, May 2017.

[46] D. Fernandez et al., "Permanent magnet temperature estimation in PM synchronous motors using low cost hall effect sensors," 2016 IEEE Energy Conversion Congress and Exposition (ECCE), Milwaukee, WI, 2016, pp. 1-8. 